\title{
MLaaS: Machine Learning as a Service
}

\author{
Mauro Ribeiro, Katarina Grolinger, Miriam A.M. Capretz \\ Department of Electrical and Computer Engineering \\ Western University, London, Ontario, Canada N6A 5B9 \\ \{mribeir5, kgroling, mcapretz\}@uwo.ca
}

\begin{abstract}
The demand for knowledge extraction has been increasing. With the growing amount of data being generated by global data sources (e.g., social media and mobile apps) and the popularization of context-specific data (e.g., the Internet of Things), companies and researchers need to connect all these data and extract valuable information. Machine learning has been gaining much attention in data mining, leveraging the birth of new solutions. This paper proposes an architecture to create a flexible and scalable machine learning as a service. An open source solution was implemented and presented. As a case study, a forecast of electricity demand was generated using real-world sensor and weather data by running different algorithms at the same time.
\end{abstract}

Keywords-Machine Learning as a Service, Supervised Learning, Regression, Prediction, Service Oriented Architecture, Service Component Architecture, Platform as a Service

\section{INTRODUCTION}

The amount of data generated has been continuously growing from global data sources like Web sites, social media, mobile applications, news networks, weather, political institutes, society and the economy. No matter how big the data are, they may be useless without proper preparation and processing. Many different machine learning algorithms have been used to extract valuable knowledge from data, e.g., for scientific modeling, consumer behavior, energy consumption forecasting, related article recommendation and user trends.

At the same time, with the popularization of sensors and mobile devices able to connect to a network (e.g., the Internet of Things), it is becoming viable to collect more data from specific contexts at higher levels of detail. By connecting global and context specific data, it is possible to extract even more detailed information and build richer knowledge using machine learning algorithms.

Large companies have enough resources to invest in their own machine learning solutions. However, small companies, developers and researchers in general have difficulties when facing the steep learning curve of how machine learning works and when building their own solutions or integrating with third-party ones. In addition, machine learning can require computational resources with impracticable costs. How could these users have access to affordable machine learning services?

One way to meet this demand is by creating a functional and ready-to-use Machine Learning as a Service (MLaaS) platform. Because multiple users will be using the same platform, computational resources can be shared or allocated on demand, reducing overall costs. By specifying a well defined interface, users can have access to machine learning process efficiently from anywhere, at any time. Users must not be concerned with implementation and computing resources, focusing mainly on the data itself.

This paper proposes a novel approach for machine learning, providing a scalable, flexible, and non-blocking platform as a service based on the service component architecture. This platform facilitates the creation, validation and execution of machine learning models. By taking advantage from service oriented architecture, the proposed approach becomes easily scalable and easy to adapt by adding, removing, changing and linking any component. This also makes the system more flexible for handling multiple data sources and different machine learning algorithms at the same time. In addition, a graphical user interface is presented to facilitate the comparison between different models.

The proposed framework source code is available ${ }^{1}$ as an open-source project to facilitate its use for various prediction modeling tasks and to enable it to be adapted for other purposes.

The following sections of this paper are organized as follows: Section II gives an overview of machine learning, service component architecture and the main related works on machine learning as a service; Section III describes the proposed architecture for MLaaS; Section IV explains the MLaaS process; Section V presents the case study; and finally, Section VI concludes the paper.

\section{RELATED WORKS}

\section{A. Machine Learning}

Machine Learning is one of the fastest growing fields in computer science [1]. It is a collection of statistical techniques for building mathematical models that can make inferences from data samples (known as a training set). Machine learning is a part of artificial intelligence: it must adapt itself to a changing environment.

Figure 1 roughly illustrates how to choose between the main categories of machine learning. There are three main types of learning [1]: (a) Supervised Learning, when the training set is labeled (i.e., it contains the attribute that the model is trying to estimate); (b) Unsupervised Learning, when the training set is not labeled, and (c) Reinforced Learning, when the learned results lead to actions that change the environment.

\footnotetext{
${ }^{1}$ https://github.com/mauro0x52/mlaas
} 


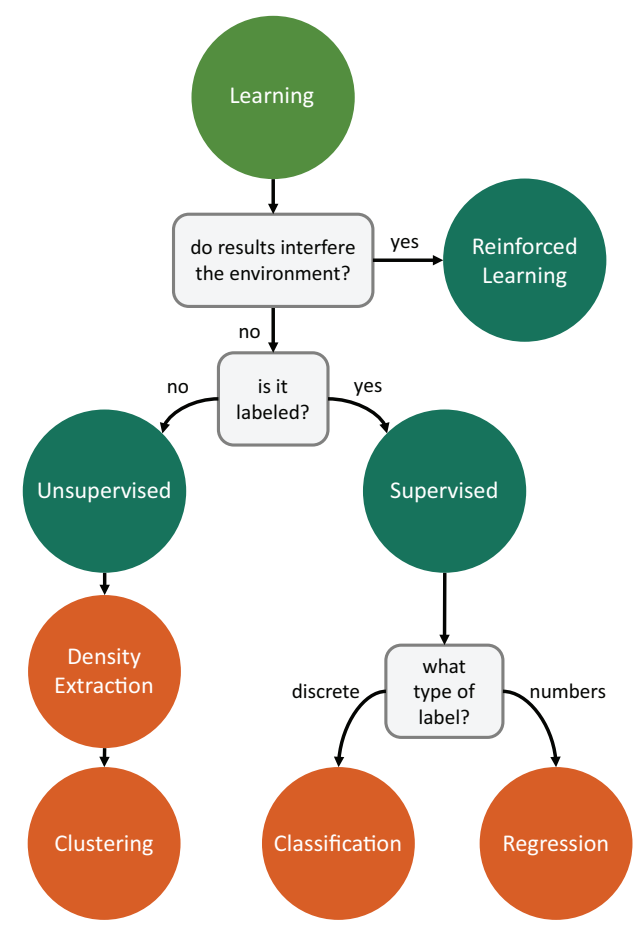

Fig. 1: Machine learning methods categorization

The labels in supervised learning can be discrete or continuous, which are handled by classification and regression algorithms respectively. Classification is used mostly for prediction, pattern recognition and outlier detection, whereas regression is used for prediction and ranking. Unsupervised learning is known as density estimation in statistics and is represented mainly by clustering algorithms. Classification, regression and clustering are widely used in data mining (applications of machine learning to large databases), whereas reinforced learning is mostly used in decision-making problems (e.g., a computer playing chess).

Independently of the applications just described, machine learning techniques work in a similar way: the model learns from a training set and then becomes able to make inferences for a new data set. This abstraction inspires the creation of a generic architecture to support any machine learning algorithm. This paper will focus on regression predictive modeling, although the approach can be adapted for other algorithms.

In predictive modeling, once rules have been extracted from past data (the training set), the model can make accurate prediction for new instances of data (the predictor set) if the future is similar to the past. Spam filtering, investment risk and energy consumption forecasting are some examples of predictive modeling. Predictive modeling approaches include: Artificial Neural Networks for energy consumption [2], Support Vector Machines for energy consumption [2] and KNearest Neighbors for wind power [3].

Validation for predictive models has a twofold importance: (a) choosing the most accurate algorithm and parameters; and (b) estimating the expected error for new predictions [1]. Ac- curacy can be related with errors, which can be calculated by comparing the estimated results from the model with the real measured results. A popular and reliable validation technique for predictive models is the K-Fold Cross-Validation. The data set is split randomly into $\mathrm{K}$ parts of the same size. One of the $\mathrm{K}$ folds is used to calculate the errors using the other $\mathrm{K}$ 1 folds to train the algorithm. The same process is repeated $\mathrm{K}$ times each time using different fold for validation. This method guarantees that the entire data set is validated with statistical significance.

Different models can perform better or worse, depending on the used algorithms, parameters and data set. However, there is no such a thing as the best learning algorithm [1]. For any algorithm, there are data sets that perform very accurately and others that perform very poorly. For the same data set, different algorithms can perform differently because of their own nature. MLaaS helps the user to run multiple algorithms and compare their performances, so the most suitable algorithm can be chosen.

\section{B. Service Component Architecture}

A service component architecture (SCA) [4] is a modeling specification for composing systems according to the principles of Service-Oriented Architecture (SOA).

SCA separates implementation concerns into three artifacts: (a) components implement its business function; (b) composites assemble various components together to create business solutions, and (c) services create an interface for remote access to component and composite functions. In a system, composites, services, and their relations with components are defined in a dynamic XML descriptor file.

Because SCA is built on top of SOA, it inherits all SOA's advantages - for example, intrinsic interoperability, inherent reuse, simplified architecture and solutions, and organizational agility [5]. In addition, whereas SOA focuses on building an architecture to design individual components, SCA focuses on assembling multiple components into a composite and facilitating design, implementation, and deployment. SCA systems have been successfully used, for example, in geographic information systems [6] and smart home systems [7] [8].

This research aims to build a platform which is capable of providing various machine learning algorithms to build different predictive models which will run at the same time. Adding a new algorithm must be simple. The system must provide well-defined APIs which can be remotely accessed over the Web by any external system. SCA provides enough artifacts to meet these requirements.

\section{Machine Learning as a Service (MLaaS)}

The increasing demand for machine learning is leveraging the emergence of new solutions. In this section, various machine learning platforms are reviewed.

PredictionIO [9] was launched in 2013. It is an open-source platform with an architecture that integrates multiple machine learning processes into a distributed and horizontally scalable 
system based on Hadoop. In addition, PredictionIO provides access through web APIs and graphical user interface (GUI).

Baldominos et al. [10] also proposed a platform built on top of Hadoop. Its implementation was capable of handling up to 30 requests at one time while maintaining a response time of less than one second.

OpenCPU [11] is another open-source platform, launched in 2014, that creates a Web API for $\mathrm{R}$ [12], a popular statistical analysis software environment. However, because it is practically a middleware for accessing $\mathrm{R}$ functions, it does not take into account many non-functional requirements like scalability and performance.

In the industry context, Google, Microsoft, and Amazon have been releasing their own proprietary platforms. Google released its Prediction API $^{2}$ in 2014. Also in 2014, Microsoft launched Azure Machine Learning ${ }^{3}$. Most recently in 2015, Amazon released AWS Machine Learning ${ }^{4}$. Their sales can prove that the demand exists. Unfortunately, the designs and implementation specifications of these products are not publicly available.

PredictionIO, OpenCPU, and Baldominos' platforms are built on top of a specific analytical tools and suffer from its restrictions. This means less flexibility for adding new machine learning algorithms, for data storage, and for deployment. Although Hadoop and $\mathrm{R}$ are open-source projects, it is not a trivial challenge to adapt them to a new approach. The same happens with the industry players and their proprietary solutions when external developers cannot have access to the code to add new algorithms.

The MLaaS proposed in this paper focuses on predictive modeling. As an architecture based on SCA specifications, the architecture facilitates the addition of new algorithms, its improvement, and its adaptation to other machine learning applications. Even the revised platforms mentioned above can be attached to proposed architecture to build prediction models.

\section{ARCHITECTURAL DESIGN}

This section describes the proposed MLaaS architecture, which is designed to support machine learning by gathering data from multiple sources and building multiple models using different algorithms. The approach focuses on predictive modeling, but it is adaptable to other applications.

The scope of this architecture deals with the machine learning itself, ignoring the front-end aspects such as the user interface. In a Model-View-Controller (MVC) perspective, this architecture focus on the model layer while the controller and view layers are only implemented as part of the case study.

The SCA diagram in Figure 2 depicts a high level overview of the architecture.

The Modeler composite is responsible for building new predictive models. A predictive model is an instance of Model$\mu$ composite, running a specific algorithm. The cardinality $0 . . \mathrm{N}$

\footnotetext{
${ }^{2}$ https://cloud.google.com/predictio

${ }^{3}$ http://azure.microsoft.com/en-us/services/machine-learning

${ }^{4}$ http://aws.amazon.com/pt/machine-learning
}

shows that MLaaS can run multiples instances of Model- $\mu$ composite at the same time, through the Build, Train, Test and Predict services. The model property shows that each instance can run with different settings.

The architecture works as follows: the Machine Learning as a Service composite receives raw data from data sources through its Send Training Set service. First, data are received and prepared by the Data Gatherer composite. The Modeler composite then receives the prepared data to train a Model$\mu$ instance. When receiving a predictor set from the Send Predictor Set service, the Model- $\mu$ instance calculates the prediction and serves it to external modules through the Get Prediction service.

The specified services provide well defined interfaces that increase the architecture's flexibility to new inputs and outputs: the Send Training Set and Send Predictor Set services enable the inclusion of various data sources that will be merged by Data Gatherer; the Build, Train, Test and Predict consumers enable the architecture to be pluggable with different Model$\mu$ instances; and the Get Report, Get Test and Get Prediction services enable different user interfaces and external systems to consume the data.

The following subsections describe each of the composites shown in Figures 2 and 3.

\section{A. Data Gatherer Composite}

The Data Gatherer composite is responsible for receiving data, pre-processing it, and feeding it to the model. One instance is created for each Send Training Set, Send Test Set or Send Predictor Set services, so that they can run in parallel and independently. The Data Gatherer composite is made up of three components arranged in a pipeline as illustrated in Figure 3; they can be described as follows:

- The Merger component merges all received data (single data points or batches) from different data sources (e.g., sensors or databases). Data sets with different schema are joined into a single multicolumn schema by related attributes (e.g., time-stamp for time-series data, categories, identifiers, etc). When finished, it forwards the data to the Outliers Remover component.

- The Outliers Remover component removes outliers (e.g., missing values, zeros, extremely high values, etc.). Once finished, it forwards the cleaned data to the Pre-Processor component.

- The Pre-Processor component modifies the data set by re-sampling, creating columns, getting the maximum, minimum, or average values, etc. When it is finished, it sends the pre-processed data to the destination component in the Modeler composite.

\section{B. Modeler Composite}

This is the core composite in the architecture, because it is responsible for building, training, testing, and running the Model- $\mu$ instances. It is made up of five components as illustrated in Figure 2, which can be described as follows: 


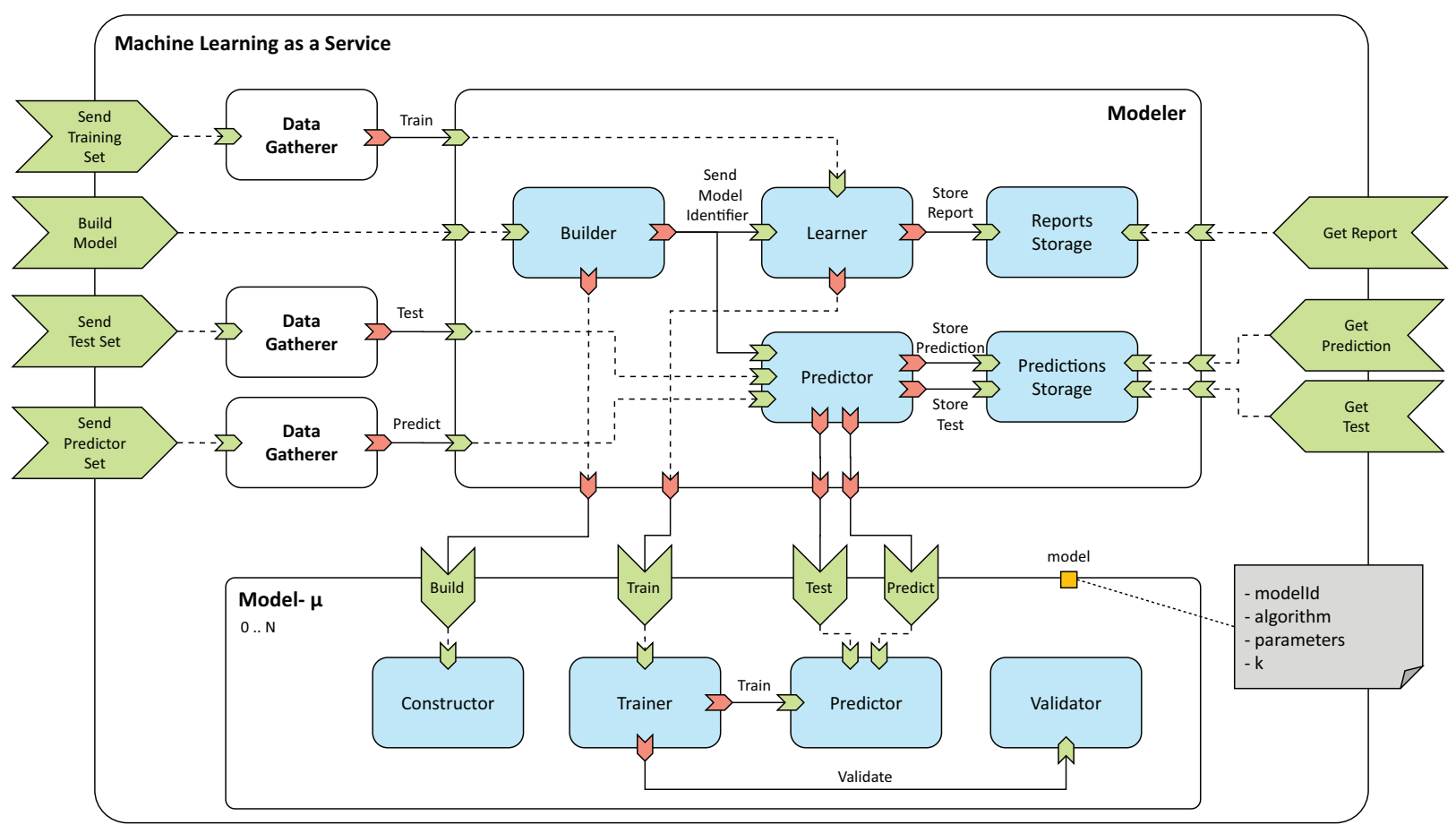

Fig. 2: MLaaS architecture using SCA notation

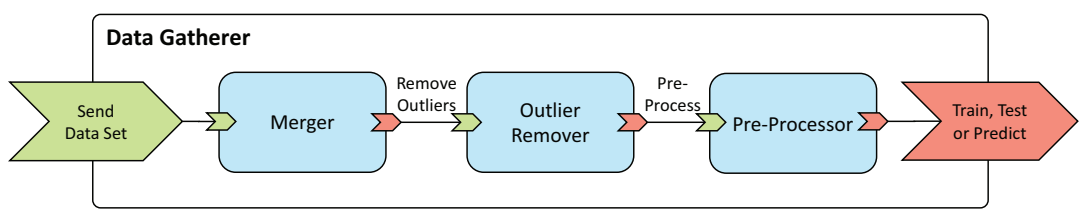

Fig. 3: Data Gatherer composite

- The Builder component receives from Build Model the parameters (e.g., algorithm and property values) to build and deploy a new model (a Model- $\mu$ instance) for the Build consumer. When the instance is created, Builder sends the model identifier back to the consumer and forwards it to the Learner and Predictor components.

- The Learner component receives the pre-processed data from the Train service and forwards them to the destined Model- $\mu$ instance. When it receives the training report from the Model- $\mu$ instance through the Train consumer callback, it forwards it to Reports Storage.

- The Reports Storage component receives the report from the Learner component through the Store Report service and serves it to external consumers through the Get Report service.

- The Predictor component receives the predictor set from the Predict service and forwards it to the Model through the Predict consumer, which will return the prediction through a callback. The prediction will be returned to the Predict requester and also forwarded to Predictions Storage. Predictor is also responsible for forwarding the testing set.

- The Predictions Storage component receives and stores the predictions and tests from the Store Prediction and Store Test services and provides them to external consumers through the Get Prediction and Get Test services.

\section{Model- $\mu$ Composite}

The Model- $\mu$ composite is an architecture for building different models. It holds all the implemented algorithms source codes (e.g., Multilayer Perceptron), but only one must be loaded. The algorithm to be loaded and its parameters should be specified when calling the Build service. In other words, for each Build Model service request, a new instance of a Model- $\mu$ composite is created.

The model property describes how the model needs to be built and executed. It is composed of four sub-properties: modelId: is the model unique identifier, algorithm: specifies which algorithm is going to be used by the model, parameters: adjust the algorithm behavior, and $k$ : the number of folds to use in the K-Fold Cross-Validation.

The Train, Test, and Predict service specifications enable the Modeler composite to interact with any Model- $\mu$ instance.

The Model- $\mu$ composite is made up of four components, which can be described as follows:

- The Constructor component is responsible for loading the right algorithm and setting the properties of the model in- 
stance using the Build service request parameters. When the instance is set up and running, it is ready to provide Train, Test and Predict services.

- The Trainer component receives the training set from the Train service and forwards it to Validator and Predictor components through the Validate and Train services respectively. When validation is finished, the Trainer component receives the validation report from the Validate service callback and returns it to the consumer through Train service callback.

- The Validator component receives the training set from the Validate service, feeds it to the model and validates the model (e.g., K-Fold Cross-Validation), returning a report.

- The Predictor component receives the training set from the Train service to feed the model for future prediction requests. When receiving predictor sets through the Predict service, it calculates and returns the predictions.

The implemented algorithms source code must be responsible only for training and predicting. Testing and validating do not depend on the algorithm itself, but on the results, which can be found by using the algorithm's training and predicting functions. Therefore, testing and validating functions are responsibilities of Validator and Predictor components, increasing standardization and reducing the effort when adding a new algorithm.

\section{MLAaS PROCESS}

The diagram in Figure 4 illustrates the main interaction flow between the Consumer, the Modeler and the Model$\mu$ composites. To simplify, the earlier stage related to the Data Gatherer composite is ignored by assuming that data have already been pre-processed. The term Consumer in the following discussion refers to a generic consumer using the Modeler component.

The main flow is divided into three stages:

- Building: it starts with the Consumer requesting the Builder component to build a new model through the Build Model service. The Builder component will then create and configure a new Model- $\mu$ instance. When the building operation is complete, the Builder component sends the new model identifier to the Learner and Predictor components and to the Consumer.

- Training: the Consumer is now able to train the instantiated model. It sends the already pre-processed training set to the Learner component through the Train service, which will forward the training set to the Trainer component of the Model- $\mu$ instance. The Trainer component will make two requests at the same time: one to the Validator component to validate the model (e.g., K-Fold Cross-Validation) and another to the Predictor component to be trained for future prediction requests. When validation is complete, the Validator component responds to Trainer component with the validation report, which contains information such as error measurements. The
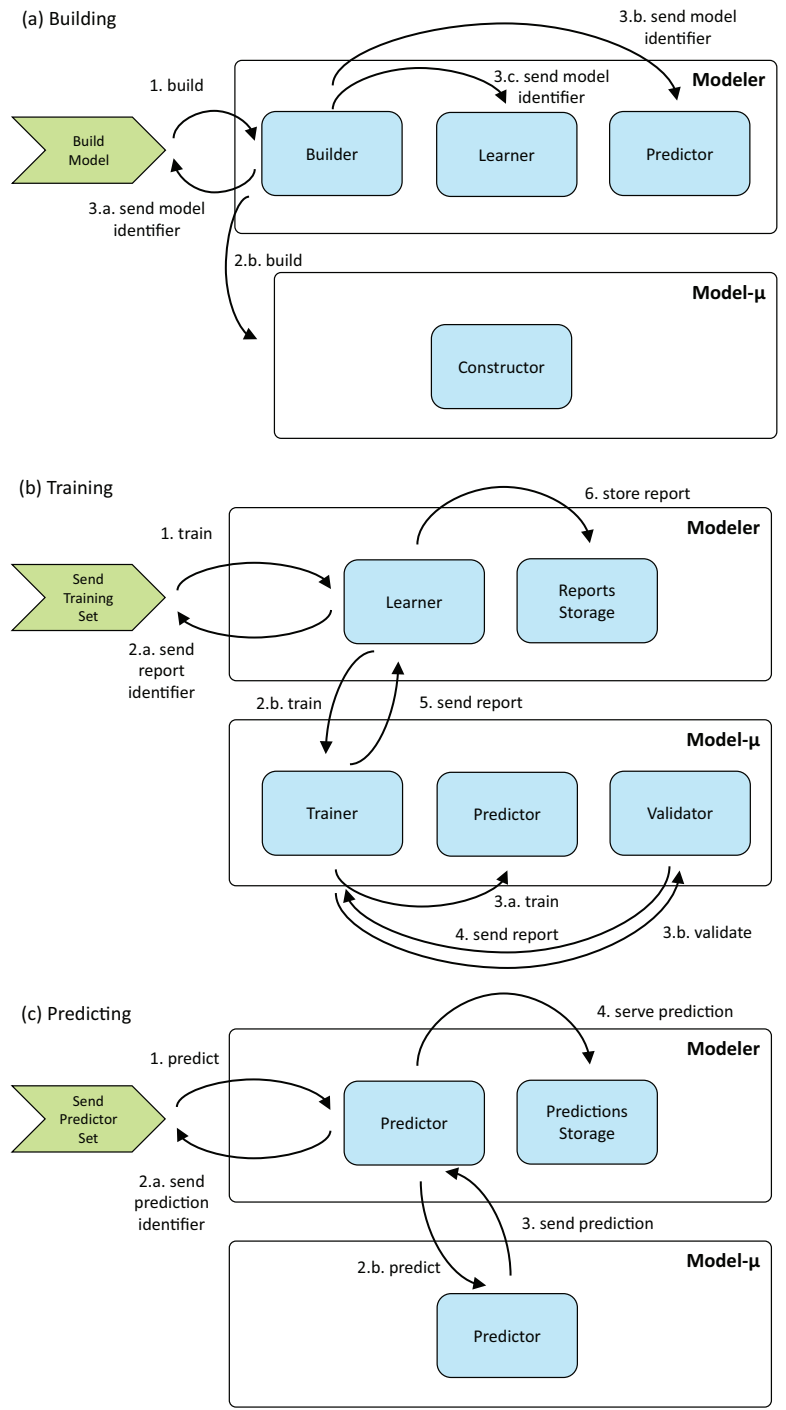

Fig. 4: Communication flow for the three machine learning stages

report will be stored into Reports Storage component for future retrievals.

- Predicting: the model is ready to predict. The Consumer sends the predictor set to the Modeler composite's Predictor component, which will forward to the Model- $\mu$ instance's Predictor component, where the prediction is calculated and returned to the Modeler. The predictions are sent to the Predictions Storage to be stored and served.

In Training and Predicting stages, the Consumer receives the report and prediction identifiers as soon as the Learner and Predictor components receive the request, so it is not necessary to keep the connection while the entire request is be processed. When the report or prediction is ready, it can be accessed from Reports Storage and Predictions Storage components, using the specific identifier.

A Training Stage can also be considered and works similarly to the Predicting Stage. The main difference is the final result, which contains testing information such as errors. 


\section{CASE Study}

The goal of this case study is to forecast energy demand based on past electricity demand data data for an office building, using different machine learning algorithms and finding the best-performing one. This experiment focuses mainly on the Modeler and Model- $\mu$ composites.

The proposed architecture was implemented using electricity demand data from Powersmiths' office building, in Brampton, ON, Canada. The data set were pre-processed before feeding them to the system. This data set was made up of 13 daily attributes: the energy demand peak, six weather attributes and six time attributes. The six weather attributes were: maximum temperature, minimum temperature, average temperature, maximum humidity, minimum humidity and average humidity. The six time attributes were: year, month (from 1 to 12), day of the month (from 1 to 31), day of the year (from 0 to 365), weekDay (from 0 for Sunday to 6 for Saturday) and dayType (0 for a business day, 1 for a weekend and 2 for a holiday).

The system was built using Node.js because of its ease and agility for coding and deploying Web services and handling JSON. Because there are currently no SCA frameworks for Node.js, one had to be implemented. JSON was used for Web service communication, data storage and the SCA artifact descriptor file. A simple user interface was developed to generate effective illustrations of the results obtained.

The source code is available in a public repository ${ }^{5}$.

\section{A. Algorithms}

To evaluate the architectural flexibility of running different machine learning models at the same time, Model- $\mu$ composite was implemented to support the following algorithms:

- Multi-Layer Perceptron (MLP): one of the most used techniques when evaluating machine learning models, and one of the most used for electrical consumption problems [2]. It was implemented using the Synaptic package ${ }^{6}$.

- Support Vector Regression (SVR): also one of the most used techniques for electrical consumption problems [2]. It was implemented using the Node-SVM package ${ }^{7}$.

- K-Nearest Neighbors (KNN): easy to understand, to code, and to debug. This algorithm was coded for this experiment.

A generic Algorithm class was coded under object-oriented programming structure, defining the standard interface for train and predict function calls. A new algorithm can be implemented simply by inheriting the Algorithm class and making minor adaptations. In this case study, the $\mathrm{KNN} \mathrm{Al-}$ gorithm class was implemented first to test and validate the Model- $\mu$ composite. Later, using the same code structure, MLP Algorithm and SVR Algorithm classes were coded and imported into Model- $\mu$ composite.

\footnotetext{
${ }^{5}$ https://github.com/mauro0x52/mlaas

${ }^{6}$ http://synaptic.juancazala.com

${ }^{7}$ https://github.com/nicolaspanel/node-svm
}

When a Model- $\mu$ instance is built, the algorithm with the parameters (both specified in the model property) is loaded.

The test and validate functions are performed by Predictor and Validation components respectively, and not by the Algorithm class. Both functions use the results from Algorithm's train and predict calls.

The Validator component implements de K-Fold CrossValidation method to validate the model, calculating the mean absolute errors and the mean square errors. The number of folds $\mathrm{K}$ can be defined to the model property when building a new model.

The architectural design and the dynamic artifacts descriptor file make it possible to create new Model- $\mu$ instances dynamically. After the new Model- $\mu$ instance is deployed and the artifacts descriptor file is updated, the new Model- $\mu$ instance will be available without the need to recompile or restart the system.

\section{B. Results}

Three different models were created by instantiating the Model- $\mu$ composite. Table I shows the parameters used for each model. The models were requested to predict using a test set, which contains all the 13 attributes including the real measured daily electricity demand peaks. For the KFold Cross-Validation, $\mathrm{K}=10$ was fixed for all the models. The models were also requested to run a prediction using a different predictor set.

Figure 5 shows a screenshot of the MLaaS graphical user interface (GUI). Through the navigation bar, the user can access models (list, create and remove), train, test and predict models and consult a graphical summary of the results. The first row of charts shows the validation performance, with three graphics showing the mean absolute errors, mean square errors, and the execution time for each of the three models. The second row shows the test performance, comparing the mean absolute errors, mean square errors, and execution time for the three models. The third row is a chart comparing the three models' test results with the real measured data from the test set. Finally, the last row shows the results of a prediction.

TABLE I: Model Parameters

\begin{tabular}{c|rl}
\hline Algorithm & Parameter & \multicolumn{1}{c}{ Value } \\
\hline \hline \multirow{2}{*}{ KNN } & $\mathrm{k}$ & 10 \\
& max distance & 2 \\
\hline \multirow{3}{*}{ MLP } & nodes per layer & $12,14,1$ \\
& learning rate & 0.1 \\
& max iterations & 1000 \\
& min error & 0.0001 \\
\hline \multirow{3}{*}{ SVR } & gamma & $0.125,0.5,1$ \\
& $\mathrm{c}$ & $8,16,32$ \\
& epsilon & $0.001,0.125,0.5$ \\
& retained variance & 0.995 \\
\hline
\end{tabular}




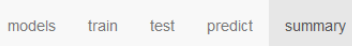

Summary
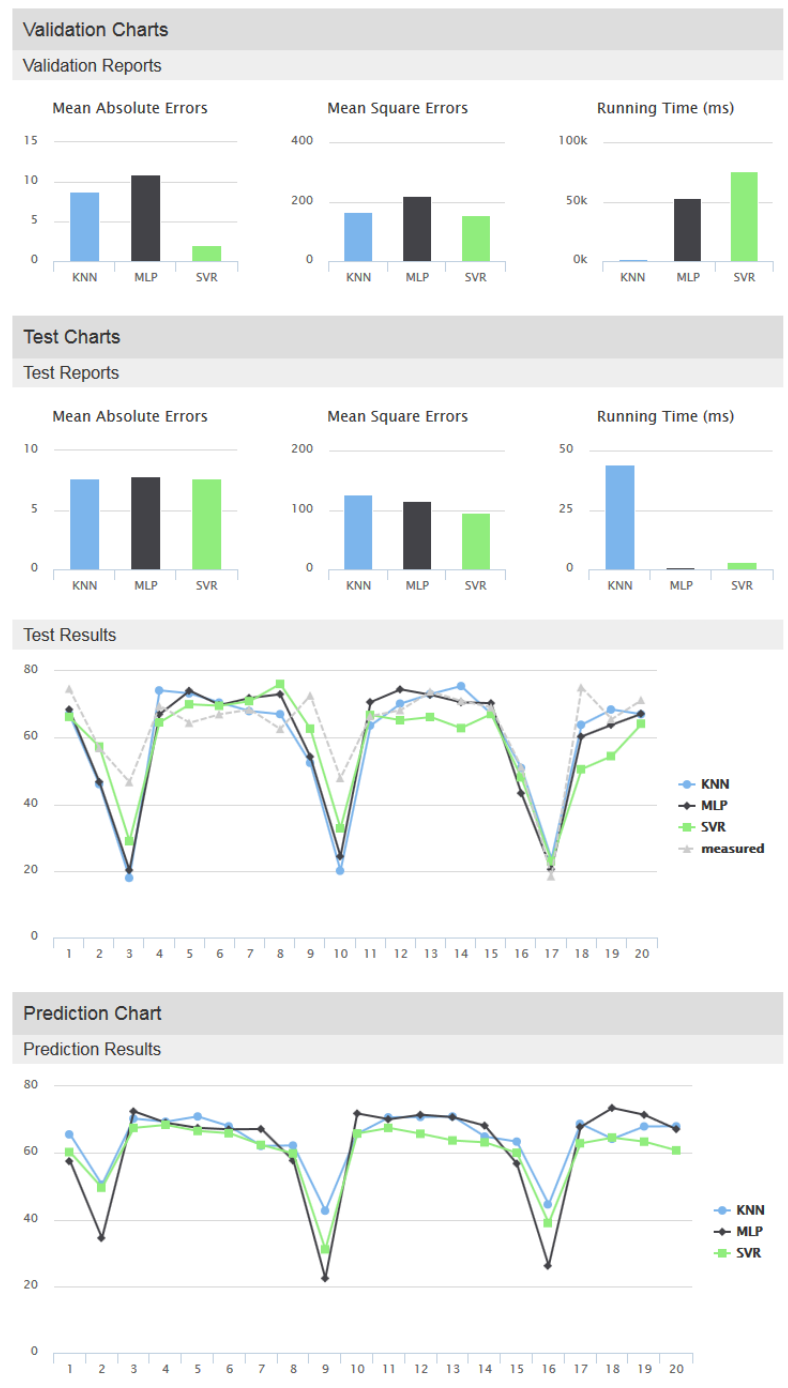

Fig. 5: MLaaS screenshot comparing KNN, MLP, and SVR.

The SVR model showed better accuracy - it had the lowest mean absolute errors and mean square errors - both in validation and in testing. Although the KNN model had better accuracy in validation than the MLP model, it had the worse mean square error in testing.

The KNN model performed much faster during validation and could finish executing even while the SVR and MLP models were still running. The SVR model finished the validation last. On the other hand, during testing, MLP model finished first and KNN model was the last. In other words, one model's processing did not block the CPU as it would have on a singlethreaded server.

\section{CONClusions}

With the growing amount of data available, companies and researchers are demanding feasible and affordable ways to extract knowledge from all this data. This paper has presented a novel architecture for a scalable, flexible, and non-blocking machine learning as a service based on SCA and focusing on predictive modeling. The proposed architecture can support multiple data sources and create various models with different algorithms, parameters, and training sets.

To prove the concept, the system was built to predict electricity demand using real-world data. Once the main architecture is working and at least one algorithm coded, it is simple to implement other algorithms. It is possible to execute multiple models concurrently.

For future research, MLaaS can be adapted to machine learning applications other than predictive modeling, for example, pattern recognition, outlier detection, ranking and clustering.

\section{ACKNOWLEDGE}

This research was supported in part by an NSERC CRD at Western University (CRDPJ 453294-13). Additionally, the authors would like to acknowledge the support provided by Powersmiths.

\section{REFERENCES}

[1] E. Alpaydin, Introduction to machine learning. MIT press, 2014.

[2] a. S. Ahmad, M. Y. Hassan, M. P. Abdullah, H. a. Rahman, F. Hussin, H. Abdullah, and R. Saidur, "A review on applications of ANN and SVM for building electrical energy consumption forecasting," Renewable and Sustainable Energy Reviews, vol. 33, pp. 102-109, 2014. [Online]. Available: http://dx.doi.org/10.1016/j.rser.2014.01.069

[3] M. Yesilbudak, S. Sagiroglu, and I. Colak, "A new approach to very short term wind speed prediction using k-nearest neighbor classification," Energy Conversion and Management, vol. 69, pp. 77-86, 2013.

[4] Service Component Architecture Assembly Model Specification Version 1.1. Accessed: 30-04-2015. [Online]. Available: http://docs.oasis-open. org/opencsa/sca-assembly/sca-assembly-1.1-spec-cd03.html

[5] T. Erl, Service-Oriented Architecture: Concepts, Technology, and Design. Pearson Education India, 2005.

[6] F.-C. Lin, L.-K. Chung, W.-Y. Ku, L.-R. Chu, and T.-Y. Chou, "Service component architecture for geographic information system in cloud computing infrastructure," in Advanced Information Networking and Applications (AINA), 2013 IEEE 27th International Conference on. IEEE, 2013, pp. 368-373.

[7] T. Calmant, J. C. Américo, D. Donsez, and O. Gattaz, "A dynamic sca-based system for smart homes and offices," in Service-Oriented Computing-ICSOC 2012 Workshops. Springer, 2013, pp. 435-438.

[8] C.-C. Lo, D.-Y. Chen, and K.-M. Chao, "Dynamic data driven smart home system based on a service component architecture," in Computer Supported Cooperative Work in Design (CSCWD), 2010 14th International Conference on. IEEE, 2010, pp. 473-478.

[9] S. Chan, T. Stone, K. P. Szeto, and K. H. Chan, "PredictionIO: a distributed machine learning server for practical software development," in Proceedings of the 22nd ACM international conference on Conference on information \& knowledge management. ACM, 2013, pp. 2493-2496.

[10] A. Baldominos, E. Albacete, Y. Saez, and P. Isasi, "A scalable machine learning online service for big data real-time analysis," in Computational Intelligence in Big Data (CIBD), 2014 IEEE Symposium on. IEEE, 2014, pp. 1-8.

[11] J. Ooms, "The OpenCPU System: Towards a Universal Interface for Scientific Computing through Separation of Concerns," arXiv:1406.4806, no. 2000, pp. 1-23, 2014. [Online]. Available: http://arxiv.org/abs/1406.4806

[12] R Core Team, R: A Language and Environment for Statistical Computing, R Foundation for Statistical Computing, Vienna, Austria, 2013, ISBN 3-900051-07-0. [Online]. Available: http://www.R-project. org/ 